\title{
MINIATURE RECONFIGURABLE ASSEMBLY LINE FOR SMALL PRODUCTS
}

\author{
Alain Codourey ${ }^{1}$, Sébastien Perroud ${ }^{1}$, Yves Mussard ${ }^{2}$ \\ ${ }^{\prime}$ CSEM Centre Suisse d'Electronique et de Microtechnique SA, Untere Gründlistrasse 1, \\ CH-6055 Alpnach Dorf. Switzerland; ${ }^{2} H T I$ Biel/Bienne, Quellgasse 21, CH-2501 Biel, \\ Switzerland.
}

Abstract: In this paper we present a new concept of miniaturized production line for the assembly of small components. The concept is based on miniature robotic cells that work together. A miniature parallel robot has been developed for this purpose and is presented in this paper.

Key words: Parallel Robot, Microfactory, Miniature Production Line, Miniature Robot

\section{INTRODUCTION}

Production of microsystems today requires usually bulky and costly machines that have disproportionate size compared to the size of the product they produce. A new concept called microfactory was introduced in the early 90 's in Japan to bring solutions to this problem. A first prototype has been presented that is able to produce micro ball bearings ${ }^{1}$. This first prototype includes several machines of small size: a micro-lathe, a milling machine, a micropress, a transfer arm and a two-fingered microhand. The control of the microfactory is simple. No automatic transfer is organized between the machines. The target device is selected with a selector and then manually controlled. Several works have already been done on global concept of the microfactory ${ }^{2}$ and on mechanical aspects. Minifactories have also been studied and prototypes have been developed by Hollis ${ }^{3}$ and Gaugel ${ }^{4}$.

In this paper, we present a new concept for the microfactory based on small production units of $1 \mathrm{dm} 3$. A special emphasis is given to the 
development of a miniature robot based on the delta parallel structure and on its control system.

\section{CONCEPT OF THE MICROFACTORY}

A new concept has been established for the design of microfactories in collaboration between CSEM, EPFL and HTI-Biel. This concept has been named pocket factory. It is based on elementary assembly units called microboxes assembled together in series so that they build up a complete factory line. Each microbox has a volume of $100 \times 100 \times 100 \mathrm{~mm}^{3}$ and contains a robot, a transfer system for the components, a feeder system and some devices for the process such as a glue dispensing system for example. In certain cases, the microbox is built as a clean room environment of class 100 . A possible layout of such an assembly line is shown in figure 1 .

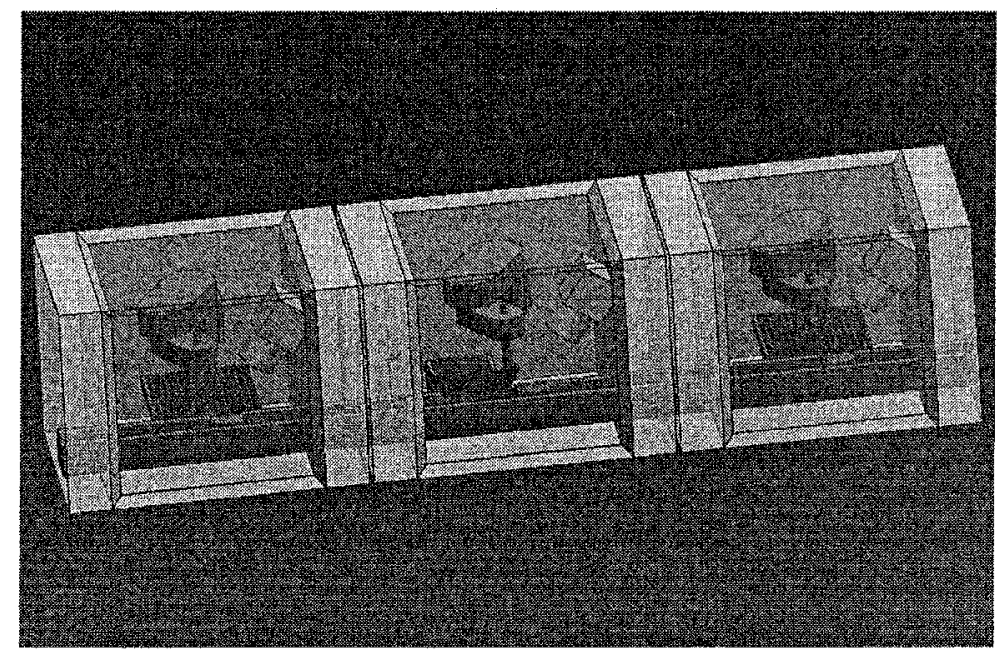

Figure 1. A possible layout of the pocket factory

Based on this idea, a fast robot with parallel structure based on the delta principle $^{5}$ has been developed. This robot is described in the next section. 


\section{MINI-DELTA ROBOT FOR THE POCKET FACTORY}

The design of miniature robots is challenging, especially because the scaling laws are not favorable to the miniaturization of motors. On the other hand, miniaturization of mechanics leads, for the same rigidity, to a much lighter structure, giving high potential to high cadency of motion. Because the motors get big in comparison to the mechanics, it is also favorable to use parallel structures, where the motors are fixed onto the base and do not move with the robot.

\subsection{Mechanical Design}

Small motors usually have very high rotational speed for very little torque, but what is required is exactly the contrary. A gear box with high reduction ratio is thus needed to achieve the desired torques. This implies unfortunately the introduction of high friction in the mechanism and very often also backlash. Thanks to the parallel structure of the delta, the size of the motors is not very important in our case. The chosen motors are placed above the microbox and the transmission of the motion to the arm is realized through a cranted belt with a reduction of $1: 2$ only. High resolution encoders with interpolation electronics are used to get the desired resolution of 0.5 $\mu \mathrm{m}$.

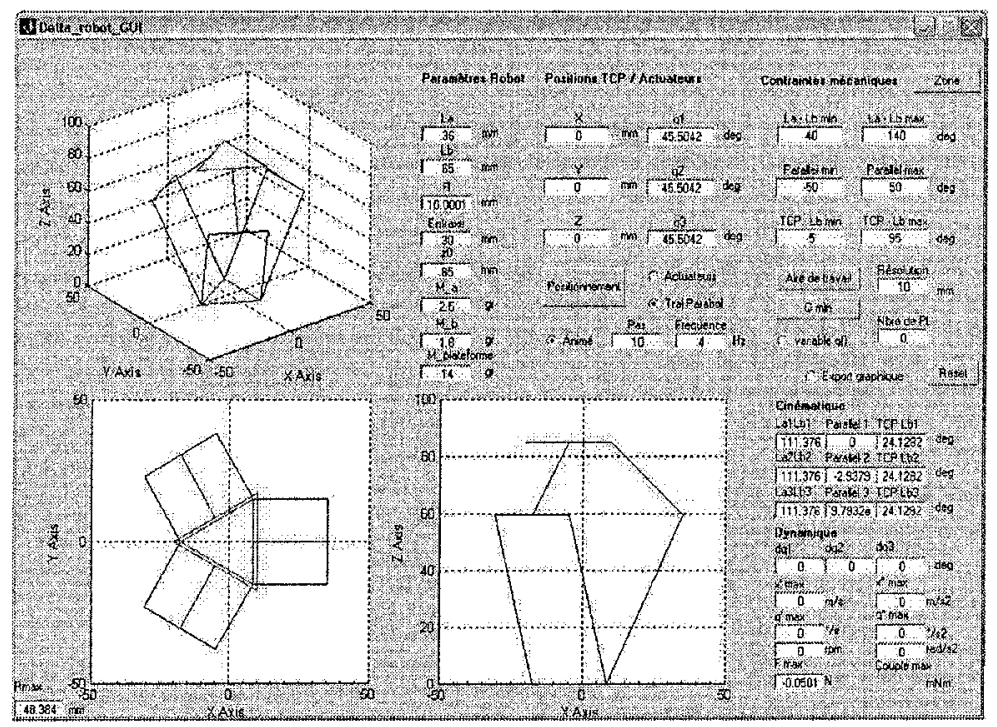

Figure 2. Simulation Environment for Optimization Purposes 


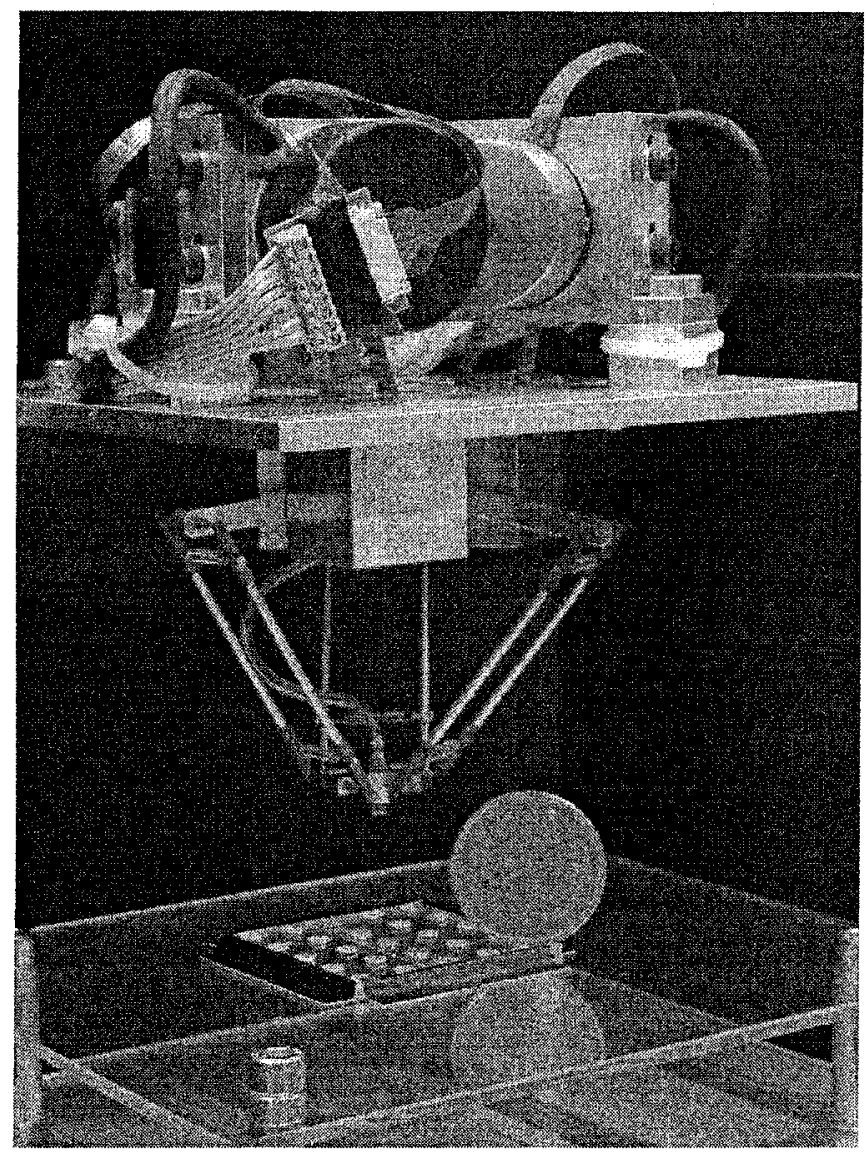

Figure 3. The optimized robot structure finally realized

The geometry of the robot has been optimized in a dedicated simulation environment (Figure 2). The optimization led to the robot shown in Figure 3, which has the characteristics given in Table 1 . 
Table 1. Main characteristics of the Pocket Delta

\begin{tabular}{ll}
\hline Feature & Values \\
\hline Size & $130 \times 130 \times 160 \mathrm{~mm}$ \\
Workspace & $60 \times 60 \times 40 \mathrm{~mm}$ \\
Moving mass & $23 \mathrm{gr}$. \\
Payload & $10 \mathrm{gr}$ \\
Repeatability & $\pm 2 \mu \mathrm{m}$ \\
Cadency & $>3$ pick \& place $/ \mathrm{s}$ \\
\hline
\end{tabular}

\subsection{Control Structure}

The controller is based on a classical PC with simple interface boards for interfacing the robot hardware (Figure 4). It might be connected to a webcam or another PC through the Ethernet interface. The control software is based on the real-time CSEM Java Framework ${ }^{6}$.

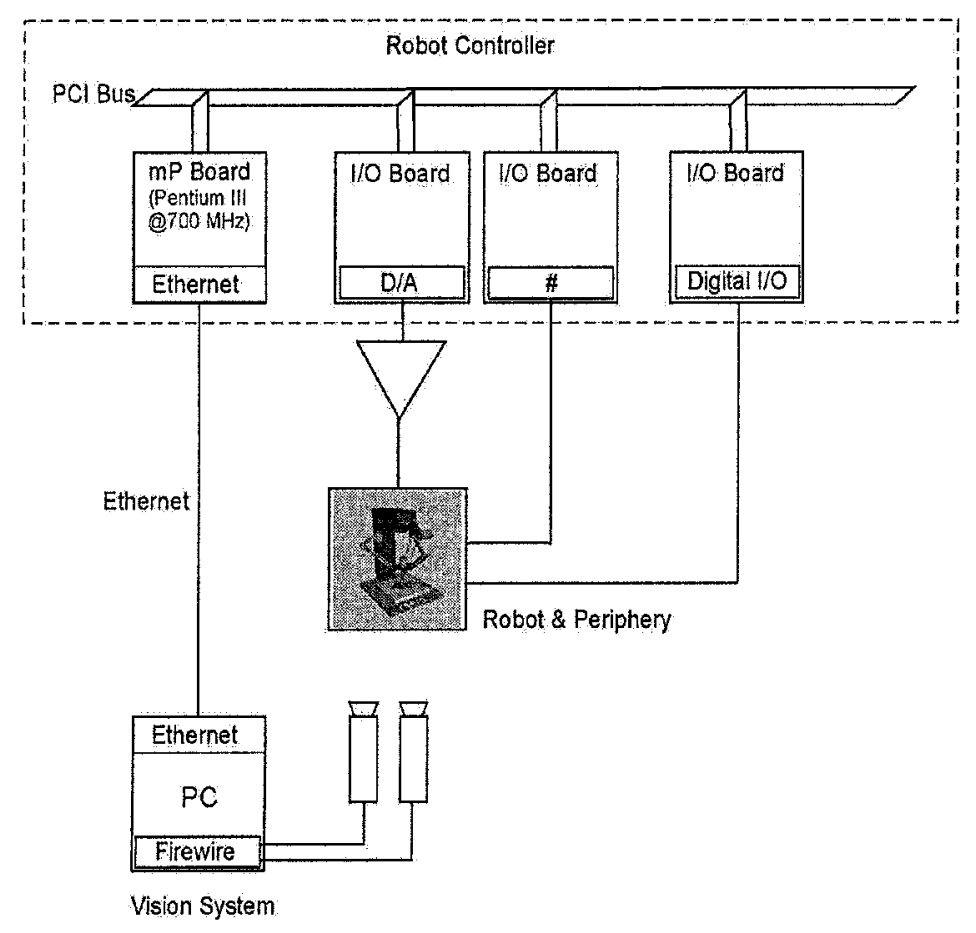

Figure 4. Control structure of the robot

This software framework has been developed at CSEM with an open design that allows extending it in any way, depending on the actual application. It is organized as a set of packages offering classes for low-level 
functions that may be useful for a wide range of technical software, and specific classes for robotics (see Figure 5). The low-level class library consists of the following components:

- A real-time thread class that can be used to install periodic, high priority tasks for control purposes.

- An embedded web-server that executes software codes within the robot controller through "virtual" cgi scripts. This enables control of the robot over a network.

- A hardware periphery abstraction layer that completely separates the application software from the actual $\mathrm{I} / \mathrm{O}$ boards. Thus, changes of the $\mathrm{I} / \mathrm{O}$ periphery only require updating a configuration file and restarting the application.

A detailed description of the framework as well as comparison of different virtual machines can be found in ${ }^{6}$.

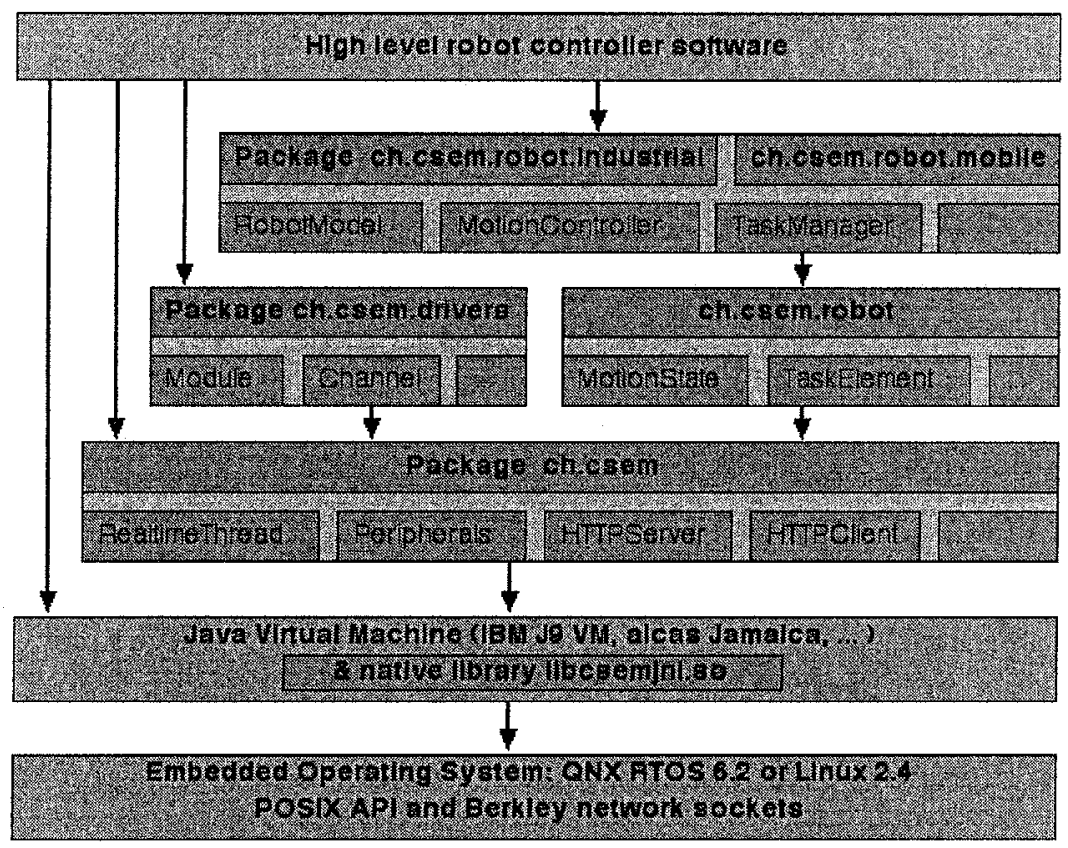

Figure 5. Software layers of the CSEM Java Framework for robot control 


\section{CONCLUSION}

The new miniature robot is fast and precise and is well suited to assembly tasks of small components inside the microfactory concept. As demonstrator, the robot was able to realize conditioning of watch's gearwheels from a vision assisted feeder into a palette. The combination of many of these small robots will lead to new concepts for the production of small parts. A typical assembly line might look like the one in Figure 6.

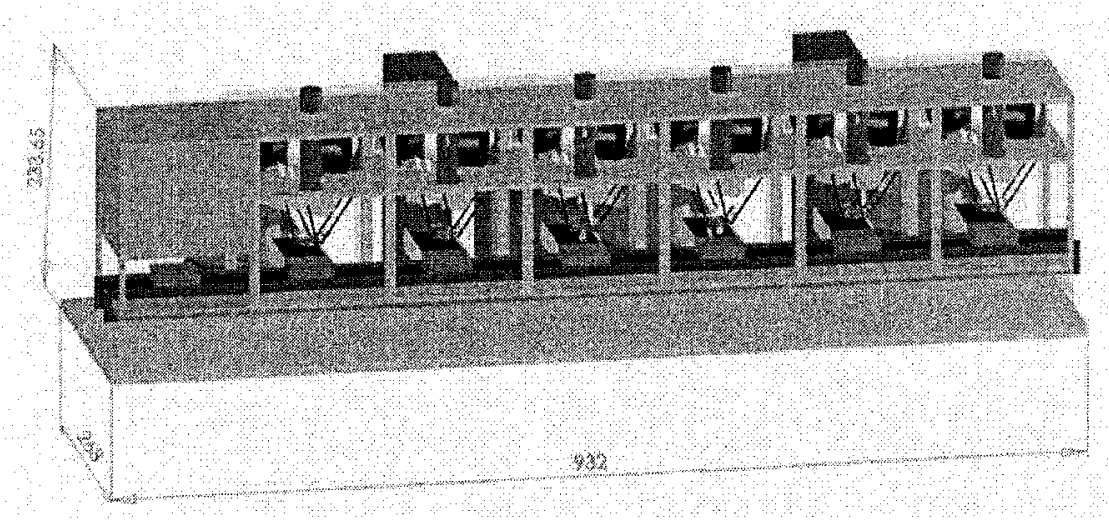

Figure 6. A pocket factory for the assembly of small electrical components. It is based on 6 PocketDelta robot, each of them realizing a single assembly task.

\section{ACKNOWLEDGMENT}

This project is supported by the cantons of central Switzerland and the MCCS Micro Center Central Switzerland. Their support is gratefully acknowledged. Part of this work has been done at EPFL and at HTI Biel. We thank them warmly for their collaboration.

\section{REFERENCES}

1. N. Ooyama et al., Desktop Machining Microfactory, Proceedings $2^{\text {nd }}$ International Workshop on Microfactories, Fribourg, Switzerland, Oct, 9-10, 2000.

2. T. Hirano et al, Industrial Impact of the Microfactory, Proceedings $2^{\text {nd }}$ International Workshop on Microfactories, Fribourg, Switzerland, Oct. 9-10, 2000. 
3. R. Hoolis and J. Gowdy, Miniature Factories for Precision Assembly, Int. Workshop on Microfactories, Tsukuba, Japan, pp. 9-14, November 1998.

4. T. Gaugel et al, Advanced modular production concept for miniaturied products, Proceedings $2^{\text {nd }}$ International Workshop on Microfactories, Fribourg, Switzerland, Oct. 9-10, 2000.

5. R. Clavel, Conception d'un robot parallèle rapide à 4 degrés de liberté, Thèse EPFL No 925, 1991.

6. M. Honegger, "A Java-Based Framework for the Design of Robot Controllers", in Proceedings of Msy'02, Winterthur, Switzerland, October 2002. 